\title{
Analysis of the fractal dimension of grain boundaries of AA7050 aluminum alloys and its relationship to fracture toughness
}

\author{
Hamideh Khanbareh • Xiaomin Wu • \\ Sybrand van der Zwaag
}

Received: 20 February 2012/Accepted: 28 April 2012/Published online: 17 May 2012

(C) The Author(s) 2012. This article is published with open access at Springerlink.com

\begin{abstract}
Quantitative analysis of the grain boundaries in partially recrystallized microstructures of heat-treated 7050 aluminum alloys has been performed. Fractal dimensions of the extracted grain boundaries were calculated by boxcounting method. Five different types of tear-tested materials rolled in different processes each at two orientations of $0^{\circ}$ and $90^{\circ}$ were studied. Efforts were made to connect the fractal dimensions of grain boundaries in the crack propagation direction to the fracture toughness (unit propagation energy, UPE, in tear test). The results show that there is a linear correlation between UPE and the fractal dimensions of the grain boundaries along the crack propagation direction for both $0^{\circ}$ and $90^{\circ}$ samples. The dependence corresponds well with the observation of transition from intergranular fracture to transgranular fracture with the increase of UPE. Quantitative analysis has also been performed on the micrographs to estimate the degree of recrystallization and the grain size in crack growth direction. No correlation between the fraction of recrystallized grains and the UPE could be detected.
\end{abstract}

\section{Introduction}

The concept of fractal geometry introduced by Mandelbrot [1] has been applied extensively to describe complex and irregular patterns in natural science. In the science of metallic materials fractal analysis has been conducted to

H. Khanbareh $(\bowtie) \cdot X$. Wu $\cdot$ S. van der Zwaag

Novel Aerospace Materials Group, Faculty of Aerospace

Engineering, Delft University of Technology, Kluyverweg 1,

2629 HS Delft, The Netherlands

e-mail: H.Khanbareh@tudelft.nl quantify the shape of various microstructural features, for instance dislocation patterns [2-4], powder particles [2], dendritic structures [2], slip lines [2], precipitates [2, 5-7], void structures $[2,7]$, fracture surfaces $[8,9]$, and grain boundaries [10-13].

In polycrystalline metallic materials the structure of grain boundaries plays an important role in defining the mechanical properties [14]. Since grain boundaries are the preferred sites for crack nucleation and growth, intergranular fracture is the main origin of brittleness of engineering materials [15]. The prevention of intergranular fracture for materials at high temperature applications can be obtained by strengthening the grain boundaries [16] by modification of the grain boundary morphology. It has been reported that cold work, hot work, or heat treatments can generate serrated grain boundaries in alloys, which improve the high temperature strength of the materials, especially the creep rupture and high temperature fatigue properties, compared to the alloys with straight grain boundaries [10, 17-20]. The effect of grain boundary serration on creep properties of metallic materials has been investigated for several systems; austenitic AISI 316 stainless steel $[11,21]$ and Ni-based and Co-based superalloys [18, 22-25]. In austenitic heat resisting steels formation of rugged grain boundaries by grain boundary reaction precipitates has been reported to improve the high temperature low cycle fatigue strength of [20]. The improved high temperature resistance of these materials is mainly attributed to reduction of grain boundary sliding [26], decrease in stress concentration at grain boundary triple points [17], crack path deflection which hinders the crack growth process $[27,28]$, and the lengthening of the fracture path [17, 28]. However, no research has been found that surveyed the effect of grain boundary serration on mechanical properties of metallic materials at room temperature. 
In this article the structure of serrated grain boundaries of heat-treated 7050 aluminum alloys rolled according to different hot rolling strategies are examined quantitatively. The term "grain boundary" refers to the grain boundary profile between recrystallized and unrecrystallized regions in two dimensional section of the microstructures [29]. Since these alloys are widely used for manufacturing the structural components of aircraft where fracture toughness and damage tolerance properties are important [30, 31], investigating the correlation between the degree of serration of the grain boundaries and fracture toughness of the materials is of scientific and technological significance.

The quantification of the ruggedness of the grain boundary can be done by determination of its fractal dimension. By definition a fractal dimension is a measure of complexity of an object and the grain boundary configuration can be estimated by the fractal dimension of grain boundary profile in two dimensional section $(1 \leq D \leq 2)$, this item is shortly called the fractal dimension of the grain boundary which has been estimated in the scale length range from $1 \mu \mathrm{m}$ to $1 \mathrm{~mm}$. Various methods are available for measuring the fractal dimension of objects. In the current work the box-counting method [1, 32-35] has been applied for fractal dimension estimation of grain boundaries, since it is easy and automatically computable for digital images of the microstructures. However, this technique needs proper implementation of image processing to extract grain boundaries. In this method meshes of increasing size cover the digital image whose fractal dimension is to be determined and number of boxes containing at least one pixel of the grain boundary are counted. The procedure starts for a box size of one pixel and is repeated for increasing box sizes, until the largest box fits the whole image. Figure 1 shows schematically the boxcounting procedure to obtain the fractal dimension of a grain boundary. The number of boxes containing at least one pixel of the grain boundary, $N(r)$, relates to the size of the boxes, $r$, following a power-law

$N(r)=N_{0} \times r^{-D}$

where $N_{0}$ is a constant. Fractal dimension $(D)$ is determined from the regression slope of the $\ln (N(r))$ versus $\ln (r)$.

The purpose of the present study is making a quantitative fractal dimension estimation on the grain boundaries of

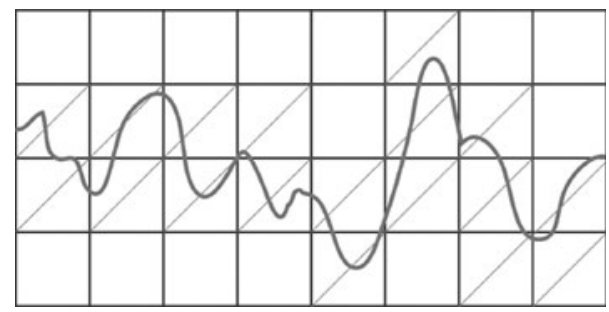

Fig. 1 Schematic illustration of box-counting method to obtain the fractal dimension of a grain boundary partially recrystallized 7050 aluminum alloys. The effect of grain boundary serration on fracture toughness of the materials is investigated.

\section{Experimental procedure}

\section{Materials}

In total five different types of AA7050 plates with identical chemical composition were received from our material supplier in the form of tear-tested samples. All plates had received $50 \%$ thickness reduction during the multipass assymetric hot rolling processes and further T74 solution treatment and artificial aging. The heat treatment yielded specimens that are partially recrystallized.

Kahn tear test is one of the most widely used methods to characterize the fracture toughness of precipitation hardening aluminum alloys in terms of the resistance of the material to tearing $[36,37]$. The tear test specimen were cut at $0^{\circ}$ and $90^{\circ}$ orientation to the rolling direction as shown in Fig. 2a. The three orthogonal characteristic directions in the rolled structures are commonly labeled as $\mathrm{L}, \mathrm{T}$, and $\mathrm{S}$, for the longitudinal (rolling direction), transverse and short transverse directions, respectively. The schematic illustration of tear test specimen

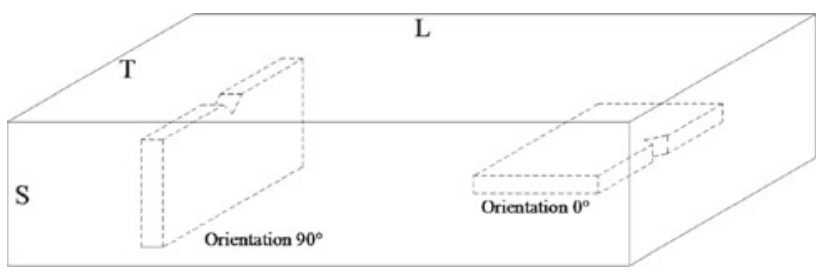

(a) Sample orientations used for fracture toughness tests of the plates.

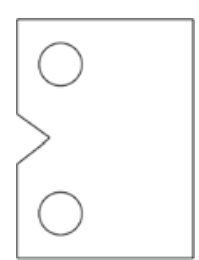

(b) un-tested tear specimen.

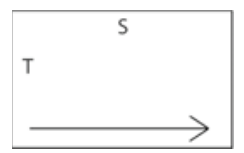

(d) S-T plane for $90^{\circ}$ samples.

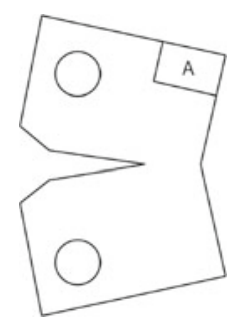

(c) Tear-tested specimen.

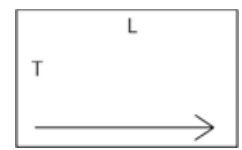

(e) L-T plane for $0^{\circ}$ samples.
Fig. 2 Schematics of sample orientations used for fracture toughness tests (a), the sketches of Kahn tear test specimens (b, c) and specimens for microstructure analysis (d, e). The specimens for microstructure analysis labeled as $A$ in c. Arrows show the crack growth direction 
used for fracture toughness measurement is shown in Fig. 2b, c. The thickness of the Kahn tear test samples was $2 \mathrm{~mm}$. The rectangular area labeled $\mathrm{A}(10 \times 15 \mathrm{~mm})$ indicates the region for microstructure analysis to reveal the undisturbed fractal dimension of the material in the final crack propagation direction. Quantitative microscopy has been carried on on S-T plane for $90^{\circ}$ orientation samples, and L-T for $0^{\circ}$ specimens (see Fig. 2d, e). In these figures arrows show the crack propagating direction on each plane. The samples for microstructure observation were cut as indicated, then mounted in an epoxy resin and ground to p4000 with sandpaper and polished with diamond suspensions to $1 / 4$ microns. For etching, a $50{ }^{\circ} \mathrm{C} 10 \%$ phosphoric acid solution was used. Samples were soaked in the solution for $4 \mathrm{~min}$ and then rinsed and dried. The microstructures of the samples were examined and analyzed at $20 \times$ magnification with an Olympus LEXT confocal microscopy, then saved as images of size $1024 \times 768$ pixels.

Image processing of microstructure image

In order to estimate fractal dimension of a grain boundary in partially recrystallized microstructures the first task is to extract the grain boundary by means of image processing techniques. An automatic image processing tool has been developed to detect the desired grain boundary. This tool operates via the following steps to extract the grain boundaries. The resulting images after each operation are shown in Fig. 3 for an example microstructure in which the grain boundary of largest recrystallized grain is to be detected:

1. Thresholding: This process transforms a gray-scale image with 256 gray levels into a binary one including pixel values of 0 and 1, black and white. The proper threshold level is defined by the user to get the desired grain boundary as a well-defined continuous profile.

2. Impurities and particles removal: To this aim the region filling operation is used. This operation identifies the pixels constituting holes within connected components in the binary image and fills them.

3. Labeling: This operation analyses an input binary image so as to identify all the connected objects in the image. In the current work, in order to preserve every structural detail of the boundaries the 8-connection concept is applied, meaning that a given foreground pixel is considered as part of the same object if it has at least one neighboring foreground pixel to the north, south, east, west, north-east, north-west, south-east or south-west of itself. A group of pixels which are all connected to each other in this way is differentiated from others by giving it a unique label.

4. Canny edge detection on extracted grain: This method produces a one pixel wide edge, while preserving the structural properties of the desired grain. (a)

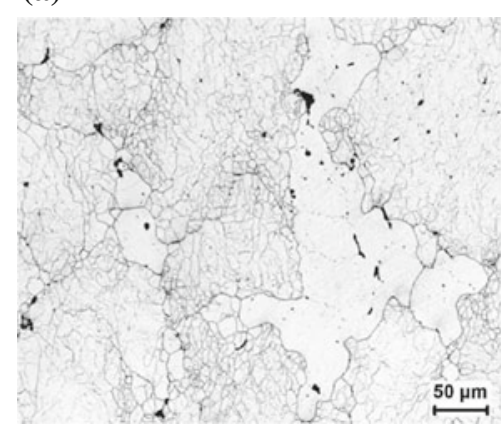

(b)

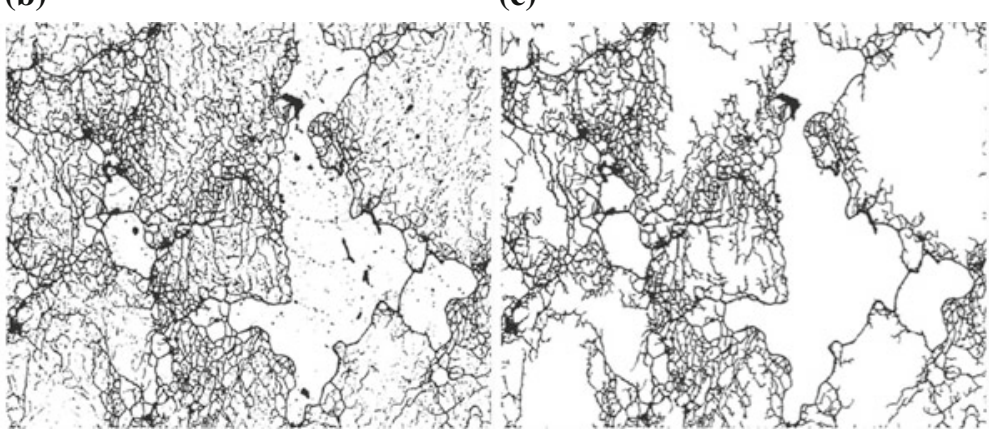

(d)

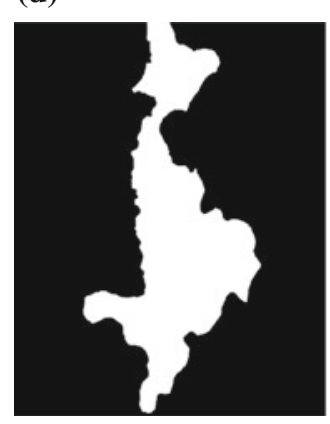

(e)

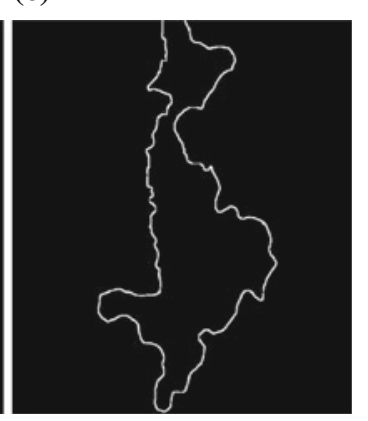

Fig. 3 Image processing procedure for an example microstructure of partially recrystallized material. a Original image, b image after thresholding, $\mathbf{c}$ image after region filling, $\mathbf{d}$ image after labeling, and $\mathbf{e}$ image after edge detection 
Since the main crack grows parallel to the $\mathrm{S}$ and $\mathrm{L}$ directions in $90^{\circ}$ and $0^{\circ}$ specimens, respectively (see Fig. 2), the fractal dimension of grain boundaries between recrystallized and unrecrystallized regions is characterized along $\mathrm{S}\left(90^{\circ}\right)$ and $\mathrm{L}\left(0^{\circ}\right)$ directions. For this purpose rectangular masks having aspect ratio of $3(300 \times 100$ pixels $)$ were located on the grain boundary, while the long axis of the box was always oriented in the crack propagation direction. Then the part of recrystallized grain boundary fitted in the mask was cut for fractal analysis. In this work 10 partial grain boundaries for each sample were chosen, cropped, and processed following the procedure described above. Box-counting measurements were then performed on the binary images of one pixel wide grain boundaries in the following manner.

\section{Box counting}

To implement the box-counting method to extracted grain boundaries a MATLAB program was developed based on the theoretical considerations discussed earlier. The original size of the images which are cropped from the micrographs according to the mask size is $300 \times 100$ pixels for the horizontal masks, and 100 pixels $\times 300$ pixels for the vertical masks. Grids of increasing size in the order of $r=1,2,4 \ldots 2^{n}$ were used to cover the image. $N$ is defined as the smallest integer such that $\max (\operatorname{size}(I)) \leq 2^{n}$, where $\max (\operatorname{size}(I))$ is the maximum dimension of the input image. In order to fulfill $\max (\operatorname{size}(I)) \leq 2^{n}$, images are padded with zeros to size $2^{9}$ over each dimension. Meshes of increasing size starting from one pixel cover the digital images and number of boxes containing at least one pixel of the grain boundary are counted. The procedure is repeated for increasing box sizes until the largest box fits the whole image and returns 1 for the box number, in this case the largest box size is 512 pixels. The fractal dimension corresponding to the slope of the $\ln (N(r))$ versus $\ln (r)$ plot is calculated by least-squares linear fitting. The program has been validated using test images of Koch curves with known fractal dimension and was found to be accurate. The indicated procedure was followed to estimate fractal dimension of 10 randomly selected grain boundaries for each studied sample. Results show that the correlation coefficients of all fitted lines show a value of more than 0.99. The overall fractal dimension for each sample is averaged over 10 test results.

\section{Results}

Figure 4a shows an example of an optical micrograph of partially recrystallized AA7050, on the metallographic plane of S-T. In the micrograph large elongated bright areas show the recrystallized regions, and the unrecrystallized grains are shown in dark gray. Constituent particles appear black and are found mostly in conjunction with the recrystallized areas. The boundaries between the recrystallized grains and un-recrystallized regions are the high angle grain boundaries on which quantitative analysis has been performed. The part of grain boundary fitted in the box has been cut as shown in Fig. $4 \mathrm{~b}$, and the resulting abstracted boundary after image processing is shown in Fig. 4c. By visually comparing Fig. 4b, c, it is obvious that within the limit of the image resolution, the detailed serrations of the grain boundaries were well captured and extracted with the image processing procedures, and are fit for fractal dimension analysis with box-counting method.

To demonstrate the effect of grain boundary morphology on on the fractal dimension analysis two extreme cases of grain boundary structure are illustrated in Fig. 5, the corresponding results of box counting for each case are also plotted. The smooth grain boundary has a fractal dimension very close to one, meaning the grain boundary is close to a one-dimensional straight line. However the highly serrated boundary shows higher fractal dimension of 1.0968

The fractal dimension for each sample averaged over ten randomly selected grain boundaries and the standard

(a)

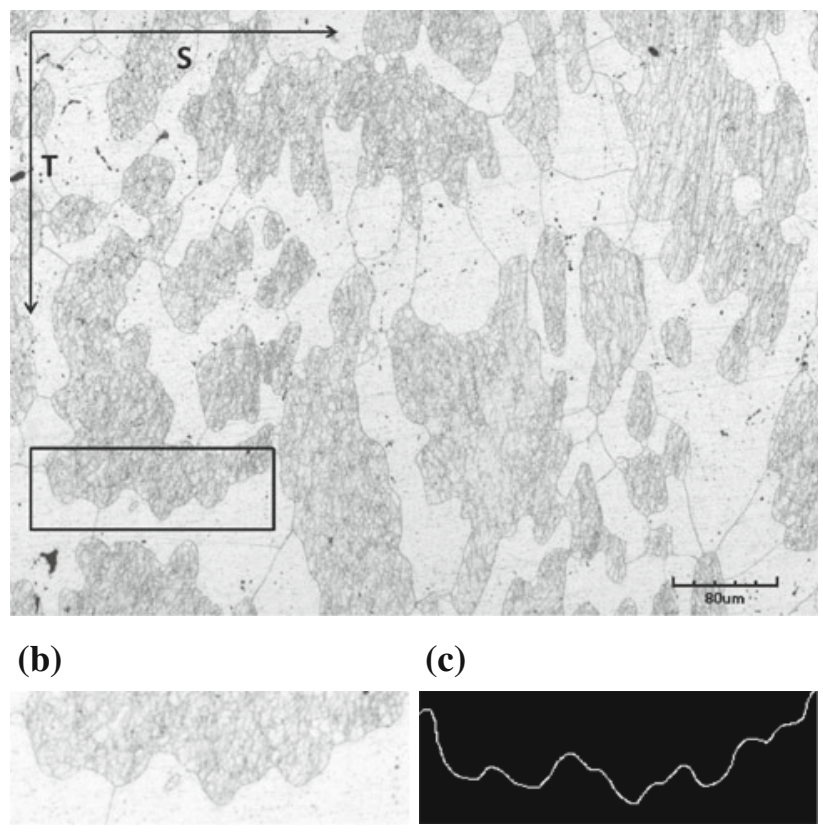

Fig. 4 Procedure of sampling the grain boundary aligned in the $S$ direction on S-T plane of $90^{\circ}$ sample. a Image of the S-T plane, the rectangular box shows the selected part of the grain boundary aligned in the $\mathrm{S}$ direction chosen for fractal analysis. b Selected boundary, $300 \times 100$ pixels. c Detected grain boundary 
(a)

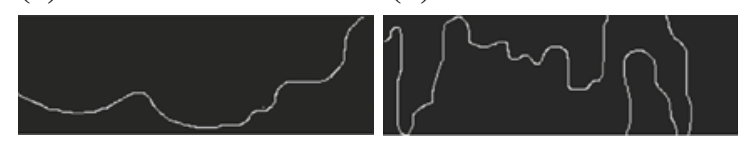

(c)

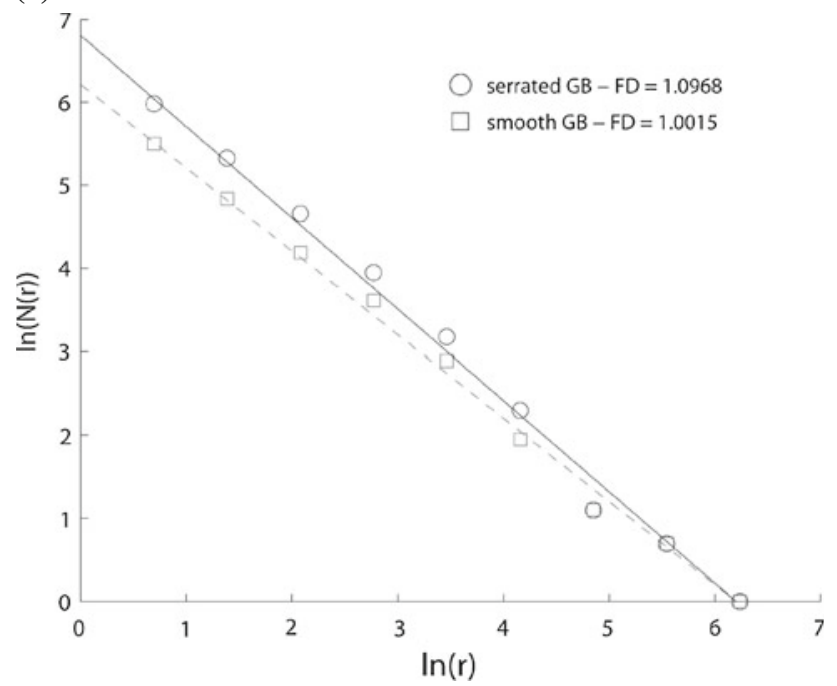

Fig. 5 Two examples of smooth and highly rugged grain boundaries. a Smooth grain boundary, FD $=1.0015$. b Serrated grain boundary, $\mathrm{FD}=1.0968$. c Box-counting results for examples of smooth and serrated grain boundaries

deviations are represented in table 1 . In each group of $0^{\circ}$ and $90^{\circ}$, sample 3 shows the highest fractal dimension and sample 4 possess the lowest fractal dimension. Comparing two different orientations of $0^{\circ}$ and $90^{\circ}$ for one rolling process, it is obvious that $90^{\circ}$ samples have higher fractal dimensions along the $\mathrm{S}$ direction than $0^{\circ}$ samples along the $\mathrm{L}$ direction.

The UPE data for every sample were averaged over three test results meeting all criteria for valid Kahn teartested load-displacement curves and the average values are shown in Table 1. Standard deviations are also reported. Samples $3-90^{\circ}$ and $4-0^{\circ}$ show the highest and the lowest UPE, respectively. For each rolling process $90^{\circ}$ samples have higher UPE values than $0^{\circ}$ samples. In the next section, the obtained results will be interpreted to justify the observed a correlation between UPE and fractal dimension of the grain boundaries.

\section{Discussion}

Effect of fractal dimension of grain boundaries on fracture toughness of the material

Investigations carried out on the fracture toughness of the 7XXX series aluminum alloys show that the fracture
Table 1 Results of box counting and UPE data

\begin{tabular}{lllll}
\hline Samples & $\begin{array}{l}\text { Average } \\
\text { FD }\end{array}$ & $\begin{array}{l}\text { FD standard } \\
\text { deviation }\end{array}$ & $\begin{array}{l}\text { Average } \\
\text { UPE }(\mathrm{KJ} / \mathrm{m} 2)\end{array}$ & $\begin{array}{l}\text { UPE standard } \\
\text { deviation }\end{array}$ \\
\hline $1-90^{\circ}$ & 1.032 & 0.012 & 76 & 11.26 \\
$2-90^{\circ}$ & 1.053 & 0.025 & 130 & 22.51 \\
$3-90^{\circ}$ & 1.094 & 0.027 & 151 & 12.01 \\
$4-90^{\circ}$ & 1.023 & 0.012 & 36 & 7.09 \\
$5-90^{\circ}$ & 1.049 & 0.023 & 119 & 17.03 \\
$1-0^{\circ}$ & 1.019 & 0.010 & 23 & 7.23 \\
$2-0^{\circ}$ & 1.023 & 0.016 & 60 & 7 \\
$3-0^{\circ}$ & 1.036 & 0.021 & 118 & 13.74 \\
$4-0^{\circ}$ & 1.018 & 0.015 & 21 & 5.03 \\
$5-0^{\circ}$ & 1.027 & 0.012 & 65 & 26.15 \\
\hline
\end{tabular}

toughness is affected by a range of microstructural features, such as coarse particles [38, 39], precipitates [40, 36], configuration of the precipitation free zone [41, 42], and grain size [43-45]. However no attempt has been made to investigate the influence of grain boundary morphology on the fracture toughness of these materials at room temperature. Since grain boundaries are the preferential path for crack propagation during fracture [14], the crack growth behavior may be affected by the shape of the boundaries. Thus the quantitative fractal dimension of grain boundaries between recrystallized and unrecrystallized regions can be used to investigate the relationship between fracture toughness and degree of serration of the grain boundaries. The low angle grain boundaries in the un-recrystallized regions are not considered in this study since they are normally very resistant to fracture $[14,46]$. Figure 6 shows the plot of UPE versus fractal dimension for both $0^{\circ}$ and $90^{\circ}$ samples. The standard deviation of UPE and fractal dimension data are shown with error bars indicating the uncertainty of the measured data. The large standard deviation of measured fractal dimensions shown on the plot implies that randomly selected grain boundaries for each sample ranges from relatively smooth to highly serrated. Still, the average fractal dimension well represents the overall structural properties of grain boundaries for each sample. The plot displays an almost linear correlation between the fractal dimension of the grain boundaries and the fracture toughness of the material for both $0^{\circ}$ and $90^{\circ}$ samples.

At low fractal dimensions the morphology of grain boundary is nearly straight, so once an intergranular crack is initiated it quickly propagates to complete fracture, resulting in low fracture toughness. This behavior is comparable to weak interface cracking observed in layered microstructure of wrought aluminum alloys when intergranular crack grows along the grain boundaries parallel to the extrusion plane resulting in low fracture toughness 
$[47,48]$. This phenomena is related to the lower strength of the grain boundaries than that of the matrix [49]. With increasing fractal dimension of a grain boundary along which the intergranular crack grows, the crack consumes more energy to propagate along the more serrated boundary, thus the fracture toughness increases. Fractographic observations revealed that the fracture mode became mixed intergranular-transgranular upon a further increase in fractal dimension of the grain boundaries. In other words, for a more serrated grain boundary the crack either grows along the rugged path of the boundary or through the matrix material where continued progression along grain boundary consumes more energy than through the matrix, both of which result in high propagation energy. This result is in agreement with the result of improved creep damage tolerance in Ni-based superalloys by introducing serrated grain boundaries [50], which has shown that the serrated grain boundaries are more resistant to intergranular cracking than the planar grain boundaries. So grain boundary strengthening by increasing the degree of irregularity of grain boundary structure results in a material with improved fracture toughness. The improved fracture toughness of the materials in the presence of serrations is attributed to delocalization of shear bands which is beneficial from a fracture energy perspective [27, 28]. It can be observed in Fig. 6 that from a fractal dimension of 1.06 onwards the fracture toughness saturates with increasing the fractal dimension of the grain boundaries, showing that the crack can not follow the highly serrated grain boundaries along the propagation direction. Hence a further increase in fractal dimension of the boundary does not affect the crack growth behavior significantly. This means that the fracture mode is mostly transgranular and controlled by the matrix material itself resulting in high fracture toughness value [49]. A schematic illustration of the

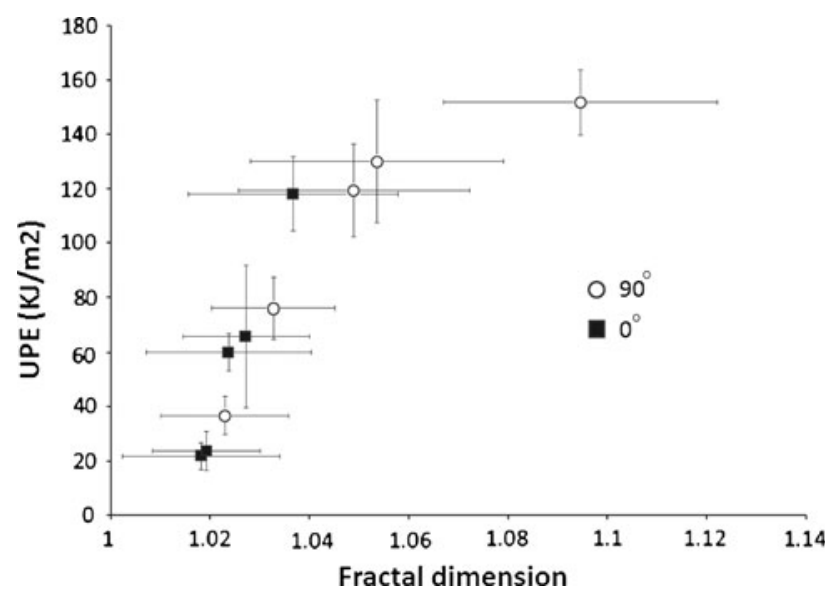

Fig. 6 Relationship between fractal dimension of the grain boundaries and UPE. Error bars show standard deviation of UPE and fractal dimensions (a)

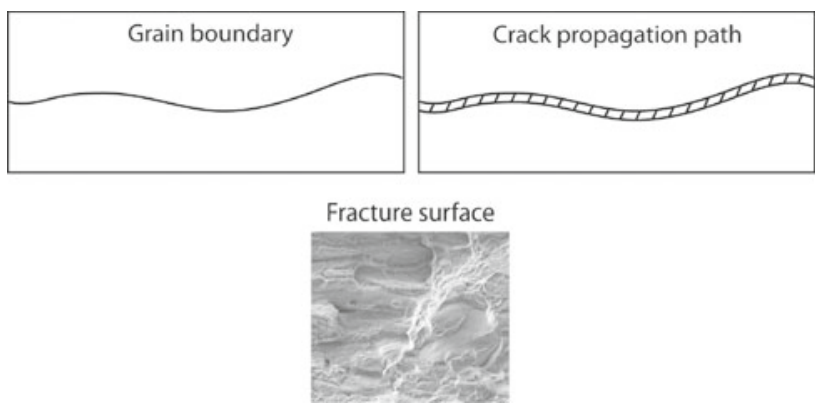

(b)

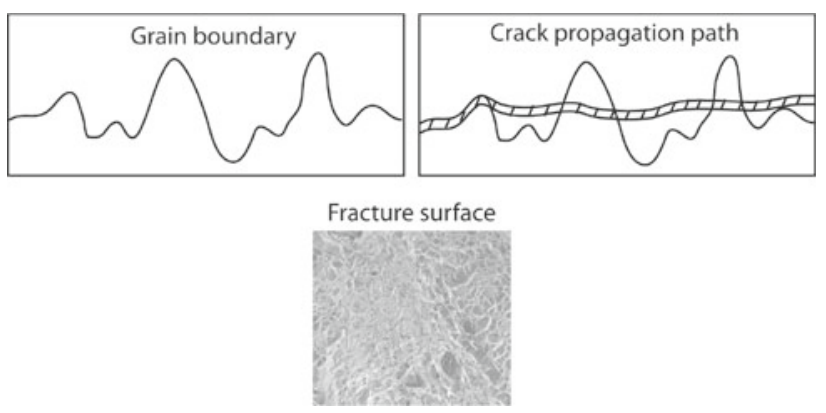

Fig. 7 Schematic illustration of crack mode for smooth and serrated grain boundaries. a Smooth grain boundary shows intergranular fracture, b serrated grain boundary shows mostly transgranular fracture

effect of grain boundary morphology on crack growth behavior is given in Fig. 7. SEM images of the intergranular and transgranular fracture modes are also provided. The above mentioned correlations are in agreement with the frequently reported relation between the fractal dimension of fracture surface profile and fracture toughness characteristics. It has been shown that the fracture surface profile of specimen with higher fracture toughness which has experienced a transgranular fracture has higher fractal dimension. However, an intergranular fracture results in a relatively flat fracture surface profile with lower fractal dimension [51-54]. Tanaka [17] also proposed a correlation between fractal dimension of the grain boundaries prior to creep deformation and the fractal dimension of the fracture surface itself; however, no investigation of fracture toughness has been reported in this case.

Effect of other microstructural features on fracture toughness of the material

In order to investigate possible relations between the fracture toughness and other attributes of the partially recrystallized microstructure of aluminum alloys, the degree of recrystallization and the recrystallized grain size in crack growth direction on crack plane has been measured for microstructures studied at a magnification of 
$20 \times$. It has been claimed that these two parameters affect fracture toughness of 7XXX series aluminum alloys [31, 44].

The plot of UPE versus degree of recrystallization is shown in Fig. 8. Measurements have been performed on five micrographs and error bars show standard deviation of data. The widespread data shows no clear relationship between UPE and degree of recrystallization for the studied materials. Further more it can be argued that the fraction of recrystallization is a non-directional property of a material and thus cannot be used to explain the large difference in fracture toughness of samples with $0^{\circ}$ and $90^{\circ}$ orientations. Therefore, the lack of correlation is to be expected.

Figure 9 shows the plot of UPE versus grain size along the crack propagation direction. which has been measured using line intercepts and averaged over ten random grains. Error bars show standard deviation of data. According to this plot a decreasing grain size in the crack growth direction increases the fracture toughness. Although flattened grains are often seen along the crack propagation direction in $90^{\circ}$ samples where higher fracture toughness was recorded compared to $0^{\circ}$ samples, the grain size itself

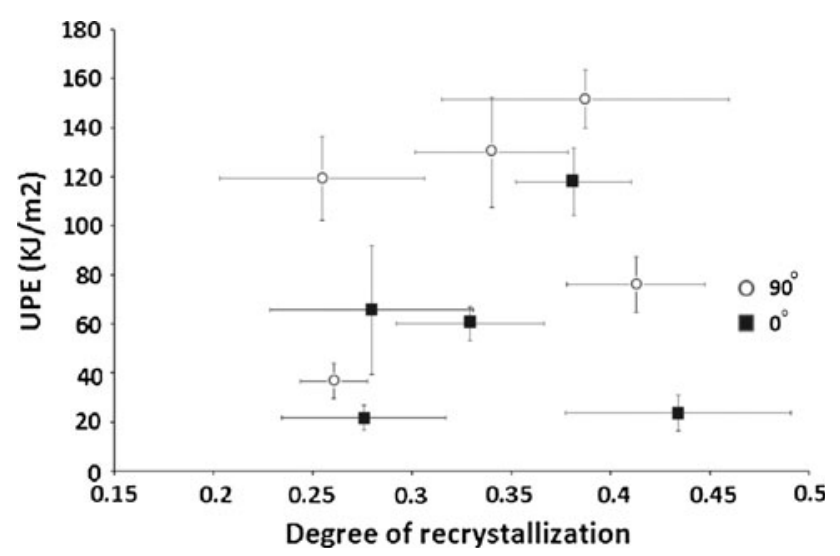

Fig. 8 Relationship between degree of recrystallization and UPE

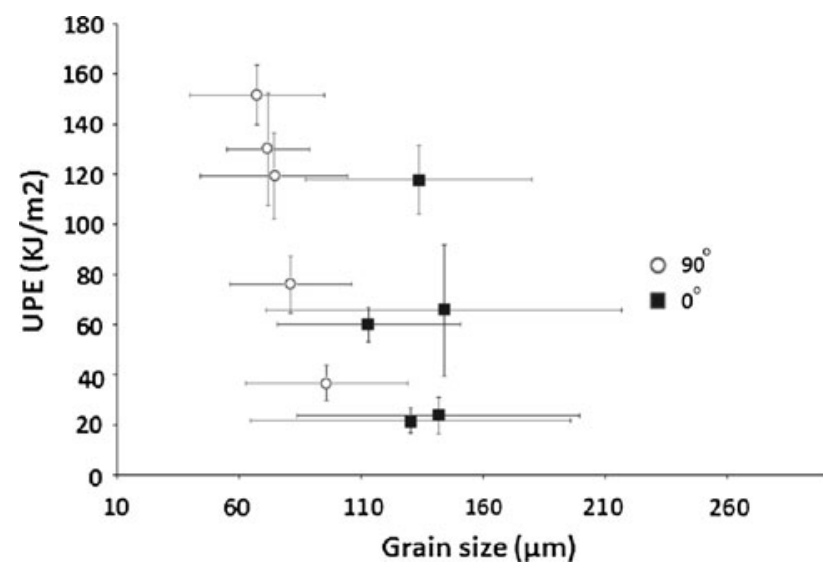

Fig. 9 Relationship between grain size and UPE is not a strong parameter to influence the fracture toughness of a material. Comparing this plot to the plot of UPE versus fractal dimension the influence of grain size appears to be of secondary importance.

\section{Conclusions}

Quantitative characterization of high angle grain boundaries carried out on Kahn tear-tested specimens has shown that there is a strong correlation between UPE data of Kahn tear test and the fractal dimension of the grain boundaries aligned in crack propagation direction calculated by boxcounting tool. Fractographic observations show that with the increase of UPE the transition takes place from intergranular fracture to transgranular fracture. Grain boundaries with low fractal dimension, thus low UPE, possess intergranular dominated fracture. Further increasing the fractal dimension shows sharp increasing of UPE with a slight increase in fractal dimensions due to higher fraction of transgranular fracture. Toward highly irregular grain boundaries the fracture mode appears to be transgranular dominated, thus not dramatically affected by fractal dimension of the grain boundaries any more. There is no clear correlation between the fraction of recrystallized grains and the UPE. The effect of the grain size in the fracture direction seems to be a secondary effect.

Acknowledgements The authors acknowledge a valuable discussion with Dr. R.C. Alderliesten on the interpretation of data. One of the authors, $\mathrm{X}$. Wu, acknowledges the financial support by the foundation Materials Innovation Institute (M2i) during the execution of the work.

Open Access This article is distributed under the terms of the Creative Commons Attribution License which permits any use, distribution, and reproduction in any medium, provided the original author(s) and the source are credited.

\section{References}

1. Mandelbrot B (1983) The fractal geometry of nature. Freeman, New York

2. Hornbogen E (1989) Int Mater Rev 34:277

3. Hahner P, Zaiser M (1999) Mater Sci Eng A 272:443

4. Zaiser M, Bay K, Hahner P (1999) Acta Mater 47:2463

5. Streitenberger P, Förster D (1991) Phys Stat Sol 164:K65

6. Laird G, Rawers J, Adams A (1992) Metall Trans A 23:2941

7. Tanaka M (1995) J Mater Sci 30:3668. doi: 10.1007/BF00351883

8. Hsiung JC and Chou YT (1998) J Mater Sci 33:2949. doi: 10.1023/A:1004302629826

9. Chang Q and Chen DL et al. (2011) J Mater Sci 46:6118. doi: 10.1007/s10853-011-5576-7

10. Hornbogen E (1987) Z Metallkd 78:622

11. Kim J, Hong H, Nam S (2009) J Nucl Mater 393:249

12. Tanaka M, Kayama A, Kato R, Ito Y (1999) Fractals 7:335 
13. Tanaka M, Kayama A, Kato R, Ito Y (1998) J Mater Sci 33:3351. doi:10.1023/A:1013241531412

14. Watanabe T, Tsurekawa S (2004) Mater Sci Eng A 387:447

15. Was GS, Bruemmer SM (1989) Mater Sci Forum 46:77

16. Watanabe T (1993) Mater Sci Eng 166:11

17. Tanaka M (1992) J Mater Sci 27:4717. doi:10.1007/BF01166012

18. Tanaka M, Iizuka H (1991) Z Metallkd 82:442

19. Huda Z, Ralph B (1990) Mater Charact 25:211

20. Iizuka H, Tanaka M (1990) J Mater Sci 25:3785. doi:10.1007/ BF00575419

21. Yamazaki M (1966) Jpn Inst Metals 30:1032

22. Larson J, Floreen S (1977) Metall Trans A 8A:51

23. Koul A, Gessinger G (1983) Acta Metall 31:1061

24. Mitchell R, Li H, Huang Z (2009) J Mater Process Technol 209: 1011

25. Henry MF, Yoo YS, Yoon DY, Choi J (1993) Metall Trans 24A: 1733

26. Tanaka M, Miyagawa O, Sakaki T, Iizuka H, Ashihara F, Fujishiro D (1988) J Mater Sci 23:621. doi:10.1007/BF01174696

27. Kitagawa H, Yuuki R, Ohira T (1975) Eng Fract Mech7:515

28. Suresh S (1983) Metall Trans A 14:2375

29. Gottstein G, Shvindlerman LS (1999) Grain boundary migration in metals: thermodynamics, kinetics, applications. CRC Press, Boca Raton

30. Heinz A, Haszler A, Keidel C, Moldenhauer S, Benedictus R, Miller W (2000) Mater Sci Eng A 280:102

31. Starke Jr EA, Staley JT (1996) Prog Aerosp Sci 32:131

32. Takayasu H (1990) Fractals in the Physical Sciences. Manchester University Press, Manchester
33. Takahashi M, Nagahama H (2003) Mater Sci Forum 426:3563

34. Takahashi M, Nagahama H (2003) Arab J Sci Eng 28:213

35. Colas R (2001) Mater Charact $46: 353$

36. Garrett G, Knott J (1978) Metall Trans A 9:1187

37. Kaufman J, Knoll A (1964) Mater Res Stand 4:151

38. Cvijović Z, Vratnica M, Rakin M (2006) Mater Sci Eng A 434:339

39. Cvijović Z, Rakin M, Vratnica M, Cvijovi I (2008) Eng Fract Mech 75:2115

40. Dumont D, Deschamps A, Brechet Y (2004) Acta Mater 52:2529

41. Pardoen T, Dumont D, Deschamps A, Brechet Y (2003) J Mech Phys Solids 51:637

42. Ryum N (1968) Acta Metall 16:327

43. Alarcon OE, Nazar AMM, Monteiro WA (1991) Mater Sci Eng A 138:275

44. Deshpande N, Gokhale A, Denzer D, Liu J (1998) Metall Mater Trans A 29:1191

45. Gokhale A, Deshpande N, Denzer D, Liu J (1998) Metall Mater Trans A 29:1203

46. Watanabe T, Tsurekawa S (1999) Acta Mater 47:4171

47. X.Li H, Xiao XR (1995) Eng Fract Mech 52:671

48. Vasudévan AK, Doherty RD (1987) Acta Metall 35:1193

49. Hornbogen E, Kreye H (1982) J Mater Sci 17:979. doi:10.1007/ BF00543516

50. Wisniewski A, Beddoes J (2009) Mater Sci Eng A 510:266

51. Lung CW, Zhang SZ (1989) Physica D 38:242

52. Tang W, Wang Y (2012) Appl Surf Sci 258:4777

53. Lung CW, and Mu ZQ (1988) Phys Rev B 38:11781

54. Bouchaud E, Lapasset G, Planes J (1990) Europhys Lett 13:73 